\title{
Longitudinal survey of Clostridium difficile presence and gut microbiota composition in a Belgian nursing home
}

\author{
Cristina Rodriguez ${ }^{1 * \dagger}$, Bernard Taminiau ${ }^{1 \dagger}$, Nicolas Korsak', Véronique Avesani ${ }^{2}$, Johan Van Broeck ${ }^{2}$, Philippe Brach ${ }^{3}$,
} Michel Delmée ${ }^{2}$ and Georges Daube ${ }^{1}$

\begin{abstract}
Background: Increasing age, several co-morbidities, environmental contamination, antibiotic exposure and other intestinal perturbations appear to be the greatest risk factors for $C$. difficile infection (CDI). Therefore, elderly care home residents are considered particularly vulnerable to the infection. The main objective of this study was to evaluate and follow the prevalence of C. difficile in 23 elderly care home residents weekly during a 4-month period. A C. difficile microbiological detection scheme was performed along with an overall microbial biodiversity study of the faeces content by 165 rRNA gene analysis.

Results: Seven out of 23 (30.4\%) residents were (at least one week) positive for C. difficile. C. difficile was detected in 14 out of 30 diarrhoeal samples (43.7\%). The most common PCR-ribotype identified was 027. MLVA showed that there was a clonal dissemination of $C$. difficile strains within the nursing home residents. 16S-profiling analyses revealed that each resident has his own bacterial imprint, which was stable during the entire study. Significant changes were observed in C. difficile positive individuals in the relative abundance of a few bacterial populations, including Lachnospiraceae and Verrucomicrobiaceae. A decrease of Akkermansia in positive subjects to the bacterium was repeatedly found.

Conclusions: A high C. difficile colonisation in nursing home residents was found, with a predominance of the hypervirulent PCR-ribotype 027 . Positive $C$. difficile status is not associated with microbiota richness or biodiversity reduction in this study. The link between Akkermansia, gut inflammation and C. difficile colonisation merits further investigations.
\end{abstract}

Keywords: C. difficile, Elderly care home residents, 165 rRNA gene analysis

\section{Background}

Clostridium difficile is a Gram-positive, anaerobic, spore-forming, rod-shaped bacterium that has been widely described in the intestinal tract of humans and animals. In 1978, C. difficile was recognized as a major cause of antibiotic associated diarrhoea and, in the most serious cases pseudomembranous colitis [1-3]. Since then, many outbreaks have been reported; most of them were associated with the emergence of a specific subtype, hyper-virulent PCR-ribotype 027 [4]. Nowadays,

\footnotetext{
* Correspondence: c.rodriguez@ulg.ac.be

${ }^{\dagger}$ Equal contributors

${ }^{1}$ Food Science Department, FARAH, Faculty of Veterinary Medicine, University of Liège, Liège, Belgium

Full list of author information is available at the end of the article
}

C. difficile is a worldwide public health concern as it is considered the major cause of antibiotic-associated infections in healthcare settings [5]. A recent report of $C$. difficile infection (CDI) cost-of-illness attributes a mean cost ranging from 8,911 to 30,049 USD for hospitalised patients (per patient/admission/episode/infection) in the USA [6] and annual economic burden estimated around 3,000 million euro in Europe [7].

CDI is more commonly diagnosed among older people in nursing homes. High isolation frequencies have been described in USA, with up to $46 \%$ of elderly residents testing positive for $C$. difficile, while in Europe or Canada the reported rates are much lower, varying between 0.8 and $10 \%$ [8]. This is partly because elderly 
people are more commonly in hospitals, have an antibiotic treatment and age-related changes in intestinal flora and host defences, as well as the presence or other underlying health problem [8-10]. These factors can have an impact on the intestinal microbiota, which may promote $C$. difficile colonisation and the development of the infection [11]. Therefore, a new concern of several studies has been the identification of the microbial communities implicated in the CDI through the use of new sequencing techniques, like metagenomics [12].

The aim of this study was to evaluate and follow the prevalence of $C$. difficile among the residents of a Belgian nursing home. Multilocus variable number of tandem repeats analysis (MLVA) was performed to determine the genetic diversity of the $C$. difficile isolates and possible cross-infection between patients. Additionally, 16S rRNA gene sequencing was used to characterise the faecal microbiota of the elderly residents, to evaluate the global evolutions of the total microbiota and to identify possible relationships between certain bacteria populations and $C$. difficile colonisation, diarrhoea and antibiotic treatment.

\section{Results}

Prevalence of $C$. difficile

A total of 242 faecal samples were collected from 23 residents in seventeen consecutive weeks (resident number 11 was excluded from the study as he finally did not agree to participate in the survey). Two subjects passed away within the four-month study period. Seven out of 23 monitored residents were positive for $C$. difficile at least once (Table 1).

There was only one case of CDI diagnosed during the study (subject 01). He was diagnosed in week eleven of

Table 1 Detailed information on 23 nursing home residents enrolled in the study, including the detection of $C$. difficile with and without enrichment

\begin{tabular}{|c|c|c|c|c|c|c|c|c|c|c|c|c|c|c|c|c|c|}
\hline \multirow{2}{*}{$\begin{array}{l}\text { Resident } \\
\text { identification }\end{array}$} & \multicolumn{17}{|c|}{ Week } \\
\hline & 1 & 2 & 3 & 4 & 5 & 6 & 7 & 8 & 9 & 10 & 11 & 12 & 13 & 14 & 15 & 16 & 17 \\
\hline 01 & $E$ & $E$ & $\neq$ & - & - & $\neq$ & - & - & $\neq$ & $E$ & $E$ & $E$ & $\neq$ & D & $\neq$ & $D$ & - \\
\hline 02 & - & - & - & - & - & - & - & - & - & - & - & - & - & - & - & - & - \\
\hline 03 & - & - & - & $\neq$ & - & - & - & - & - & $\neq$ & - & - & $\neq$ & - & $\neq$ & $\neq$ & $\neq$ \\
\hline 04 & - & - & - & - & - & - & - & - & - & - & - & $\neq$ & - & - & - & - & - \\
\hline 05 & - & - & - & $\neq$ & $\neq$ & - & $\neq$ & $\neq$ & - & - & - & - & $\neq$ & - & - & - & $\neq$ \\
\hline 06 & - & - & - & $\neq$ & - & $\neq$ & - & - & - & - & $\neq$ & - & $\neq$ & $\neq$ & - & - & $\neq$ \\
\hline 07 & - & - & - & $\neq$ & $\neq$ & - & - & - & - & $\neq$ & - & - & - & - & $\neq$ & - & $\neq$ \\
\hline 08 & $\neq$ & - & - & $\mathrm{H}$ & $\mathrm{H}$ & $\mathrm{H}$ & $\mathrm{H}$ & - & $\neq$ & - & - & - & - & $\neq$ & - & $\neq$ & $\neq$ \\
\hline 09 & - & $\neq$ & $\neq$ & - & $\neq$ & $\neq$ & $\neq$ & - & - & - & $\neq$ & - & - & - & $\neq$ & - & - \\
\hline 10 & - & $\neq$ & - & $\neq$ & - & - & $\neq$ & - & - & - & - & - & $\neq$ & - & - & $\neq$ & $\neq$ \\
\hline 12 & - & - & - & - & $\neq$ & - & - & - & $\neq$ & - & - & - & - & - & $\neq$ & - & - \\
\hline 13 & D & D & - & - & $\neq$ & - & - & - & $\neq$ & - & - & - & $\neq$ & - & $\neq$ & $\neq$ & $\neq$ \\
\hline 14 & - & - & - & - & $\neq$ & $\neq$ & $\neq$ & - & - & - & $\neq$ & - & $\neq$ & - & $\neq$ & $\neq$ & $\neq$ \\
\hline 15 & $E$ & $E$ & $\mathrm{E}$ & - & $\neq$ & $D$ & $D$ & $D$ & $D$ & $D$ & $D$ & $D$ & $D$ & $D$ & $\neq$ & $\neq$ & $D$ \\
\hline 16 & - & - & - & - & - & - & $\dagger$ & $\dagger$ & $\dagger$ & $\dagger$ & $\dagger$ & $\dagger$ & $\dagger$ & $\dagger$ & $\dagger$ & $\dagger$ & $\dagger$ \\
\hline 17 & - & - & - & $\neq$ & - & - & - & $D$ & - & - & - & - & $\neq$ & - & $\neq$ & $\neq$ & $\neq$ \\
\hline 18 & $\neq$ & $\neq$ & $\neq$ & $\neq$ & $D$ & $\neq$ & $D$ & $\neq$ & $D$ & $\neq$ & $\neq$ & $D$ & $\neq$ & $\neq$ & $\mathrm{E}$ & $\neq$ & $\mathrm{E}$ \\
\hline 19 & $\mathrm{E}$ & $E$ & $\mathrm{E}$ & $\neq$ & $\neq$ & $\neq$ & $D$ & $\neq$ & - & - & $\mathrm{E}$ & $\neq$ & $D$ & $\neq$ & - & $\neq$ & $\neq$ \\
\hline 20 & - & $\neq$ & - & $\neq$ & $\neq$ & - & $\neq$ & $\neq$ & - & $\neq$ & $\neq$ & $\neq$ & $\neq$ & $\neq$ & - & $\neq$ & $\neq$ \\
\hline 21 & $\neq$ & - & - & - & - & - & $\neq$ & - & $\neq$ & - & - & $\neq$ & $\neq$ & $\neq$ & - & $\neq$ & - \\
\hline 22 & - & - & - & - & - & - & $\dagger$ & $\dagger$ & $\dagger$ & $\dagger$ & $\dagger$ & $\dagger$ & $\dagger$ & $\dagger$ & $\dagger$ & $\dagger$ & $\dagger$ \\
\hline 23 & - & $\neq$ & - & - & - & $\neq$ & - & - & - & - & - & - & - & $\neq$ & - & - & - \\
\hline 24 & $\neq$ & $\neq$ & $D$ & $\neq$ & $\mathrm{E}$ & - & $\neq$ & $E$ & $\neq$ & - & $\neq$ & - & $\neq$ & - & $\neq$ & $\neq$ & - \\
\hline
\end{tabular}

Resident number 11 was excluded from the study

D: Positive results detected without enrichment

E: Positive results detected after 3 days of enrichment

-: Negative results for $C$. difficile presence

‡: Sample was not available

$\mathrm{H}$ : resident hospitalized

t: The resident passed away during the study period 
the study after suffer more than three episodes consecutives of diarrhoea. C. difficile was detected in 14 out of 30 diarrhoeal samples (43.7\%). Regarding the antimicrobial therapy, a total of five residents tested positive for $C$. difficile had previously received an antibiotic medication. Probiotic treatment was noted in 4 residents, two of them were positive for $C$. difficile. Only one resident (number 08) was hospitalized during the study (Table 2).

\section{Characterization of $C$. difficile isolates}

Four different PCR-ribotypes (UCL16a, UCL36, UCL46 and 027) were identified among the 38 isolates. In one resident (number 19), different PCR-ribotypes were found in different sampling days while in another subject (number 17) two different PCR-ribotypes were detected in the same sampling day (direct culture: PCR-ribotype 027; 3 days of stool enrichment: PCR-ribotype UCL36). Only in one resident (number 015), all but one samples obtained were positive for $C$. difficile and the isolated strains were all identified as PCR-ribotype 027. Three out of these four different PCR-ribotypes had toxin activity. All toxigenic isolates encoded toxin A and B, while PCR-ribotype 027 also contained the binary toxin. In addition, all types 027 contained an 18-base pair deletion, a deletion at 117 of the $t c d C$ gen and gyrA mutation associated with moxifloxacin resistance (Table 2).

\section{C. difficile MLVA analysis}

MLVA was performed in order to provide further insight into the clonal relatedness of the $C$. difficile isolates and cross-infection between patients. A total 59 isolates were obtained during the study. Among them, 44 toxigenic and non-toxigenic isolates were further analysed by MLVA. Selection of these strains was based on the inclusion of a representative number of isolates from each classified PCR ribotype. In order to determine if the seven variable-number-tandem repeat (VNTR) loci were stable over time or if subjects harboured more than one C. difficile type, isolates obtained from the same resident on direct culture and after 3 enrichment days and on different weeks were also studied by MLVA. Thirty-one different MLVA profiles were identified. However, a high degree of genetic relatedness was observed among most of the strains with the same PCR-ribotype (summed tandem repeat difference at all loci $\leq 2$ ). The C6 and A6 were the most diverse VNTR loci. Regarding the strains identified as PCR-ribotype 027, most of them were closely related. Furthermore, several isolates from patients 15, 18 and 19 had an identical MLVA profile (Table 3).

\section{Analysis of the residents' faecal microbiota by barcoded pyrosequencing}

Among the 23 residents, all available faecal samples from 13 residents $(6 \mathrm{C}$. difficile negative and 7 C. difficile positive, in total 118 faecal samples) were selected for $16 \mathrm{~S}$ profiling of their faecal microbiota. A total of 433,815 final reads were attributed to 3,940 species level OTUs (operational taxon units) among 118 samples (Additional file 1). The analysis showed that the major phyla found in patients were Firmicutes and Bacteroidetes followed by the Verrucomicrobia and the Proteobacteria (Fig. 1). On the family level, the major populations were consistent with previous human studies, Bacteroidaceae, Ruminococcaceae and Lachnospiraceae being dominant. The Verrucomicrobiaceae, Porphyromonadaceae and Rikenellaceae were subdominant (Fig. 1). The 6 major genera were Bacteroides, Akkermansia, Parabacteroides, Alistipes and two populations undefined at the genus level belonging respectively to the Lachnospiraceae and the Ruminococcaceae (Additional file 2).

The mean alpha diversity and richness was variable between residents (Additional file 3), though no resident mean values are statistically different from the rest of the cohort (Fig. 2). Moreover, the analysis of the microbiota species structure and composition showed that each patient has his own microbiological imprint during the study as revealed by weighted UNIFRAC analysis of phylogenetic distribution of the samples based on a Bray-curtis distance matrix (Fig. 2 and Additional file 4).

Among the 118 samples, 24 samples were detected positive for $C$. difficile by 16S rRNA gene analysis (Fig. 3). Reads sharing minimum $99 \%$ of identity to the $C$. difficile 16S rRNA sequence were identified as $C$. difficile. Nearest known species (Clostridium glycolicum, Terrisporobacter mayombei and Romboutsia lituseburensis) share less than $99 \%$ of nucleotide identity on the V1-V3 hypervariable region with $C$. difficile $16 \mathrm{~S}$ rRNA sequence. Thirty-seven out of 118 samples analysed by $16 \mathrm{~S}$ rRNA gene analysis were positive for $C$. difficile positive by classical microbiology. Among the positive samples, 18 samples were detected by both methods, 19 samples were positive only by culture and 6 were positive only by $16 \mathrm{~S}$ rRNA profiling.

\section{Link between $C$. difficile colonisation and faecal microbiota}

In order to explore the link between $C$. difficile colonisation and the resident microbiota, residents negative and positive for $C$. difficile were grouped. As the interindividual variability is the main driving factor for the sample clustering, the grouping was made by resident instead of strict positive and negative samples. Figure 4 shows the major mean genus relative abundance for both groups. Statistical analysis revealed that only four 
Table 2 Clinical characteristics of the 23 residents enrolled in the study and molecular type of the isolates

\begin{tabular}{|c|c|c|c|c|c|c|c|c|c|c|c|c|c|c|c|c|}
\hline $\begin{array}{l}\text { Resident } \\
\text { number }\end{array}$ & Age (years) & Genre & Status & $\begin{array}{l}\text { Room } \\
\text { floor }\end{array}$ & Diarrhea & $\begin{array}{l}\text { Hospital } \\
\text { stay }\end{array}$ & $\begin{array}{l}\text { Antibiotic } \\
\text { treatment }\end{array}$ & $\begin{array}{l}\text { Probiotic } \\
\text { treatment }\end{array}$ & C. difficile culture & PCR-ribotype & $N^{\circ}$ isolates & CE & $t c d A t c d B$ & $c d t A c d t B$ & tcdC MUT & gyrA MUT \\
\hline 01 & 77 & M & SD & 1 & + & - & + & - & + & UCL16a & 9 & + & + & - & - & - \\
\hline 02 & 78 & $\mathrm{~F}$ & D & 2 & - & - & - & - & - & - & - & - & - & - & - & - \\
\hline 03 & 92 & $\mathrm{~F}$ & D & 2 & - & - & - & - & - & - & - & - & - & - & - & - \\
\hline 04 & 88 & $\mathrm{~F}$ & D & 1 & + & - & - & - & - & - & - & - & - & - & - & - \\
\hline 05 & 93 & $\mathrm{~F}$ & D & 1 & + & - & + & + & - & - & - & - & - & - & - & - \\
\hline 06 & 86 & $\mathrm{~F}$ & D & 2 & - & - & + & - & - & - & - & - & - & - & - & - \\
\hline 07 & 92 & $F$ & SD & 3 & - & - & + & - & - & - & - & - & - & - & - & - \\
\hline 08 & 91 & $\mathrm{~F}$ & SD & 3 & - & + & + & + & - & - & - & - & - & - & - & - \\
\hline 09 & 88 & $\mathrm{~F}$ & D & 2 & - & - & - & - & - & - & - & - & - & - & - & - \\
\hline 10 & 78 & $F$ & SD & 2 & - & - & - & - & - & - & - & - & - & - & - & - \\
\hline 12 & 87 & $\mathrm{~F}$ & D & 2 & + & - & - & - & - & - & - & - & - & - & - & - \\
\hline 13 & 65 & M & D & 1 & - & - & + & - & + & UCL36 & 4 & - & - & - & - & - \\
\hline 14 & 76 & $F$ & SD & 1 & - & - & - & - & - & - & - & - & - & - & - & - \\
\hline 15 & 50 & $\mathrm{~F}$ & D & 2 & + & - & - & + & + & 027 & 22 & + & + & + & + & + \\
\hline 16 & 94 & $\mathrm{~F}$ & D & 3 & + & - & + & - & - & - & - & - & - & - & - & - \\
\hline \multirow[t]{2}{*}{17} & 63 & $F$ & D & 3 & + & - & + & - & + & UCL36 & 1 & - & - & - & - & - \\
\hline & & & & & & & & & + & 027 & 1 & + & + & + & + & + \\
\hline 18 & 86 & M & D & 2 & + & - & - & - & + & 027 & 10 & + & + & + & + & + \\
\hline \multirow[t]{3}{*}{19} & 89 & $\mathrm{~F}$ & SD & 3 & + & - & + & - & + & UCL36 & 2 & - & - & - & - & - \\
\hline & & & & & & & & & & UCL46 & 2 & + & + & - & - & - \\
\hline & & & & & & & & & & 027 & 4 & + & + & + & + & + \\
\hline 20 & 81 & $\mathrm{~F}$ & SD & 1 & - & - & + & - & - & - & & - & - & - & - & - \\
\hline 21 & 82 & $\mathrm{~F}$ & D & 1 & + & - & - & - & - & - & & - & - & - & - & - \\
\hline 22 & 83 & $\mathrm{~F}$ & D & 1 & - & - & - & - & - & - & & - & - & - & - & - \\
\hline 23 & 88 & $\mathrm{~F}$ & D & 2 & - & - & - & - & - & - & & - & - & - & - & - \\
\hline 24 & 81 & F & $D$ & 1 & - & - & + & + & + & UCL36 & 4 & - & - & - & - & - \\
\hline
\end{tabular}

$M$ male

$F$ female

SD semi-dependant residents

$D$ dependant residents

CE cytotoxicity assay using MRC- 5 cells

tcdC MUT: Presence of deletions in the regulator gene $t c d C$ (118 bp-39 bp-17 bp)

gyrA MUT: Presence of mutation in the gyrA gene associated with moxifloxacin resistance 
Table 3 MLVA profile of the isolates obtained from each nursing home resident

\begin{tabular}{|c|c|c|c|c|c|c|c|c|c|c|c|}
\hline \multirow{2}{*}{$\begin{array}{l}\text { PCR- } \\
\text { Ribotype }\end{array}$} & \multicolumn{8}{|c|}{ MLVA profile } & \multirow{2}{*}{$\begin{array}{l}\text { Resident } \\
\text { number }\end{array}$} & \multirow{2}{*}{$\begin{array}{l}\text { No. of } \\
\text { isolates }\end{array}$} & \multirow[t]{2}{*}{ Week } \\
\hline & A6 & B7 & $\mathrm{C} 6$ & E7 & G8 & CDR5 & CDR60 & $\overline{\text { Total }}$ & & & \\
\hline \multirow[t]{17}{*}{027} & 22 & 9 & 38 & 10 & 17.5 & 3.9 & 7.2 & 107.6 & 15 & 4 & $1^{\mathrm{E}} 2^{\mathrm{E}} 6^{\mathrm{E}} 7^{\mathrm{E}}$ \\
\hline & & & & & & & & & 18 & 1 & $5^{\mathrm{E}}$ \\
\hline & 23 & 9 & 38 & 10 & 17.5 & 3.9 & 7.2 & 108.6 & 15 & 1 & $3^{\mathrm{E}}$ \\
\hline & 22 & 9 & 39 & 10 & 17.5 & 3.9 & 7.2 & 108.6 & 15 & 1 & $7^{D}$ \\
\hline & 22.2 & 9 & 37.8 & 10 & 17.5 & 3.9 & 7.2 & 107.6 & 15 & 3 & $9^{\mathrm{D}} 10^{\mathrm{E}} 14^{\mathrm{E}}$ \\
\hline & & & & & & & & & 18 & 1 & $15^{\mathrm{E}}$ \\
\hline & & & & & & & & & 19 & 1 & $12^{\mathrm{D}}$ \\
\hline & 22.2 & 9 & 37.8 & 10 & 17.5 & 3.8 & 7.2 & 107.5 & 15 & 1 & $8^{\mathrm{E}}$ \\
\hline & 22 & 9 & 37.8 & 10 & 17.5 & 3.8 & 7.2 & 107.3 & 15 & 1 & $9^{\mathrm{E}}$ \\
\hline & 23 & 9 & 37.8 & 10 & 17.5 & 3.8 & 7.2 & 108.3 & 15 & 1 & $12^{\mathrm{D}}$ \\
\hline & 22.2 & 9 & 26.5 & 10 & 18.5 & 3.9 & 7.2 & 97.3 & 15 & 1 & $12^{\mathrm{E}}$ \\
\hline & 23 & 9 & 40 & 10 & 17.5 & 3.9 & 7.1 & 110.5 & 18 & 1 & $2^{\mathrm{E}}$ \\
\hline & 22 & 9 & 36.8 & 10 & 17.5 & 3.9 & 7.2 & 106.4 & 18 & 1 & $7^{D}$ \\
\hline & 22.2 & 9 & 37.8 & 10 & 17.6 & 3.9 & 7.2 & 107.7 & 18 & 1 & $7^{\mathrm{E}}$ \\
\hline & 23 & 9 & 36.8 & 10 & 17.5 & 3.8 & 7.2 & 107.3 & 18 & 1 & $9^{\mathrm{E}}$ \\
\hline & 22 & 9 & 37 & 10 & 17.5 & 3.9 & 7.2 & 106.6 & 19 & 1 & $3^{E}$ \\
\hline & 22.2 & 9 & 36.8 & 10 & 17.5 & 3.8 & 7.2 & 106.5 & 19 & 2 & $7^{\mathrm{E}} 11^{\mathrm{E}}$ \\
\hline \multirow[t]{6}{*}{ UCL16a } & 30.8 & 14.1 & $23.5^{\mathrm{a}}$ & 5 & 10.8 & 6.8 & 3.2 & 94.2 & 1 & 1 & $1^{\mathrm{E}}$ \\
\hline & 30.8 & 14 & 23.5 & 5 & 10.8 & 6.8 & 3.2 & 94.1 & 1 & 2 & $2^{\mathrm{E}} 16^{\mathrm{E}}$ \\
\hline & 30.8 & 14 & 24.5 & 5 & 10.8 & 6.8 & 3.2 & 95.1 & 1 & 1 & $10^{\mathrm{E}}$ \\
\hline & $30.7^{\mathrm{b}}$ & $14^{c}$ & 11.3 & 5 & 10.8 & 6.8 & 3.2 & 81.8 & 1 & 1 & $11^{\mathrm{E}}$ \\
\hline & 29.8 & 14 & 23.5 & 5 & 10.8 & 6.8 & 3.2 & 93.1 & 1 & 2 & $12^{\mathrm{E}} 16^{\mathrm{D}}$ \\
\hline & $31.8^{d}$ & 14 & 23.5 & 5 & 10.8 & 6.8 & 3.2 & 95.1 & 1 & 1 & $14^{\mathrm{D}}$ \\
\hline \multirow[t]{2}{*}{ UCL46 } & 28.8 & 21.1 & 22.3 & 14 & 8 & 8.8 & 2.2 & 105.2 & 19 & 1 & $1^{\mathrm{E}}$ \\
\hline & 28.8 & 21.1 & 22.5 & 14 & 8 & 8.8 & 2.3 & 105.5 & 19 & 1 & $2^{\mathrm{E}}$ \\
\hline \multirow[t]{9}{*}{ UCL36 } & 19.2 & 17 & 42.8 & 8 & 9.9 & 4.9 & 10.2 & 112 & 13 & 1 & $1^{\mathrm{D}}$ \\
\hline & 18.3 & 16 & 42.8 & 8 & 9.9 & 4.9 & 10.2 & $110^{\mathrm{e}}$ & 13 & 1 & $1^{\mathrm{E}}$ \\
\hline & 18.3 & 16 & 36.8 & 8 & 9.9 & 4.9 & 10.2 & 104.1 & 13 & 1 & $2^{D}$ \\
\hline & 19.2 & $16.1^{f}$ & 41.8 & 8 & 9.9 & 4.9 & 10.2 & 110.1 & 13 & 1 & $2^{\mathrm{E}}$ \\
\hline & 30.8 & 17 & 34.7 & 8 & 10.8 & 4.9 & 10.2 & 117.4 & 17 & 1 & $8^{\mathrm{E}}$ \\
\hline & 31.8 & 17.1 & 34.7 & 8 & 10.8 & 4.9 & 10.2 & 118.5 & 19 & 1 & $7^{D}$ \\
\hline & 31.8 & 17 & 34.8 & 8 & 10.8 & 4.9 & 10.2 & 118.5 & 24 & 1 & $3^{D}$ \\
\hline & 31.8 & 17 & 34.7 & 8 & 10.8 & 4.9 & 10.2 & 118.4 & 24 & 1 & $5^{\mathrm{E}}$ \\
\hline & 31.8 & $18.1^{9}$ & 35.8 & 8 & 10.8 & 4.9 & 10.2 & 120.6 & 24 & 1 & $8^{\mathrm{E}}$ \\
\hline
\end{tabular}

Differences found in the results after one or more repetitions: ${ }^{\mathrm{a}} 24.5 ;{ }^{\mathrm{b}} 30.8 ;{ }^{\mathrm{c}} 17.3 ;{ }^{\mathrm{d}} 26.9 ;{ }^{\mathrm{e}} 10.3 ;{ }^{\mathrm{f}} 16 ;{ }^{\mathrm{g}} 18$

${ }^{E}$ Strain isolated after 3 days of feces enrichment

D Strain isolated after direct culture of the feces

genus populations have significant relative abundance between both groups (Fig. 4). Blautia (Firmicutes) and Flavonifractor (Firmicutes) and the Lachnospiraceae_unclassified (Firmicutes) appeared more abundant in the $C$. difficile positive group, whereas Akkermansia (Verrucomicrobiaceae) abundance was higher in the $C$. difficile negative group. In order to better understand these differences, both groups were further divided into diarrhoeic ( $>1$ diarrhoeic faeces sample) or non-diarrhoeic residents. Results showed a decrease in Verrucomicrobiaceae linked to $C$. difficile positive groups (data not shown). In addition, a higher abundance of Lachnospiraceae family was detected in $C$. difficile positive diarrhoeic residents compared to other groups $(p<0.05)$ (Additional file 5). 

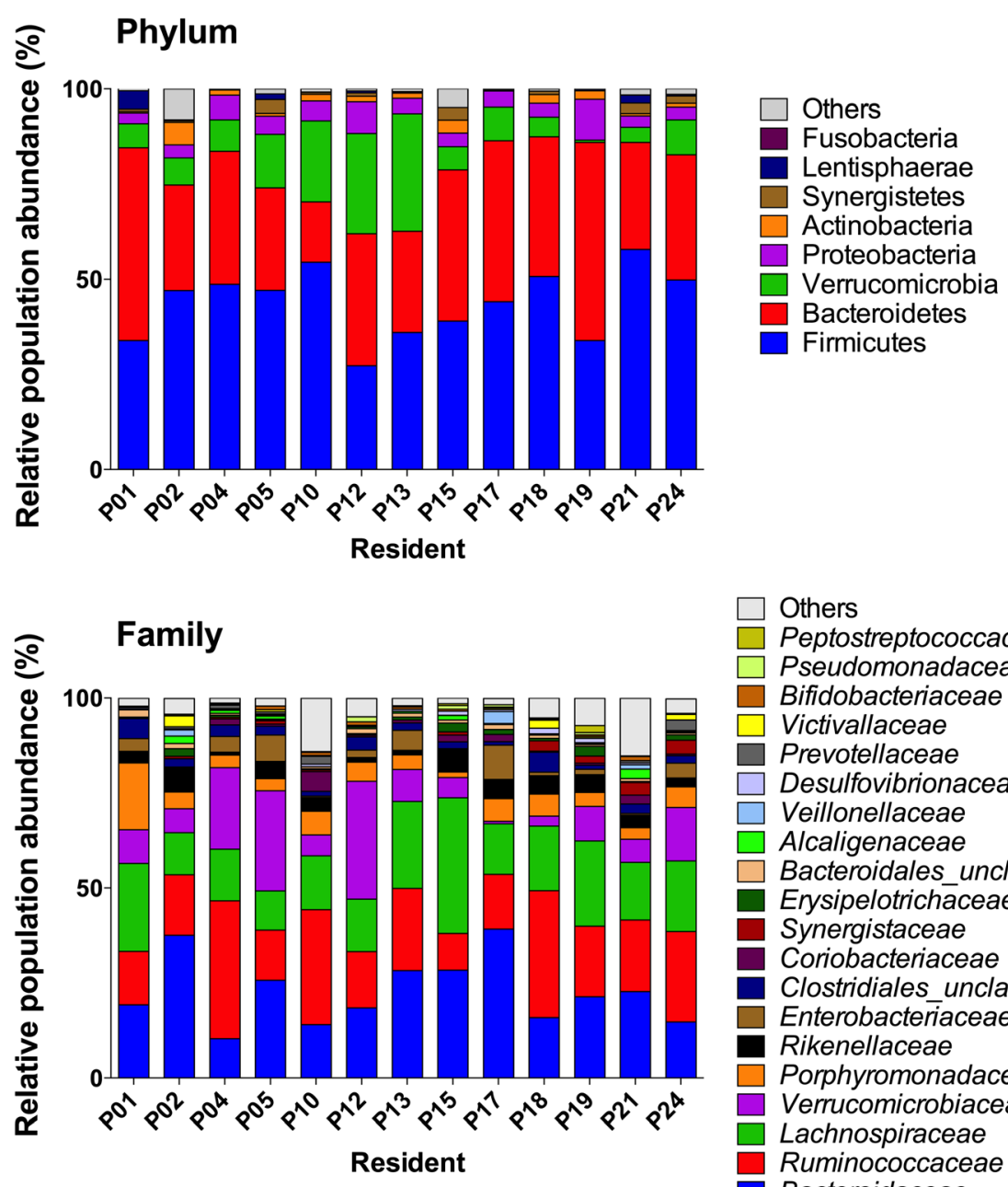

Others

Peptostreptococcaceae

Pseudomonadaceae

Bifidobacteriaceae

Victivallaceae

Prevotellaceae

Desulfovibrionaceae

Veillonellaceae

Alcaligenaceae

Bacteroidales_unclassified

- Erysipelotrichāceae

Synergistaceae

Coriobacteriaceae

Clostridiales unclassified

Enterobacteriaceae

Rikenellaceae

Porphyromonadaceae

Verrucomicrobiaceae

Lachnospiraceae

Ruminococcaceae

- Bacteroidaceae

Fig. 1 Taxonomical distribution deduced by $16 \mathrm{~S}$ rRNA profiling. Bart chart detailing the mean cumulated relative abundance of the major phyla and families for each resident

The analysis of mean alpha diversity of both groups showed that $C$. difficile positive group does not exhibited an increased faecal microbial diversity compared to $C$. difficile negative group after Bonferroni corrections (Fig. 4). No differences were revealed in the species richness (Chao index). The diarrhoeic status does not appear to have an influence on the results obtained (data not shown).

\section{Discussion}

The gut microbiota ecosystem plays a critical role in resistance to colonisation by pathogenic organisms, infection and recurrence [11]. C. difficile colonisation has been described as ten times higher in elderly nursing home residents than in the general population living outside long-term care facilities [13]. The deteriorating health status of nursing home residents, their frequent hospitalisation and the cohabitation in the same contaminated environment promote bacterial colonisation and dissemination [14, 15]. The aim of this study was to evaluate the presence of $C$. difficile in a short cohort of elderly nursing home residents and to evaluate the global evolutions of their faecal microbiota.

In the present study, $30.4 \%(7 / 23)$ were positive to $C$. difficile. In previous studies conducted in Germany, United Kingdom, Ireland, Australia or Canada, the prevalence of positive residents reported ranges between 0.80 and $10 \%$ [13, 16-19]. This prevalence is much higher in other reports in USA, varying between 6.4 and $54.8 \%$. The same scenario was reported for the incidence of CDI in Belgian hospitals when compared with other hospitals in Europe and USA [20, 21]. In this study, only one resident was diagnosed with a CDI. However, other residents presented symptoms (diarrhoea) and either stool test positive for toxigenic $C$. difficile. Therefore, the lack of clinical diagnosis or request do not exclude that other residents suffered CDI during the study period. On the other hand, positive residents 


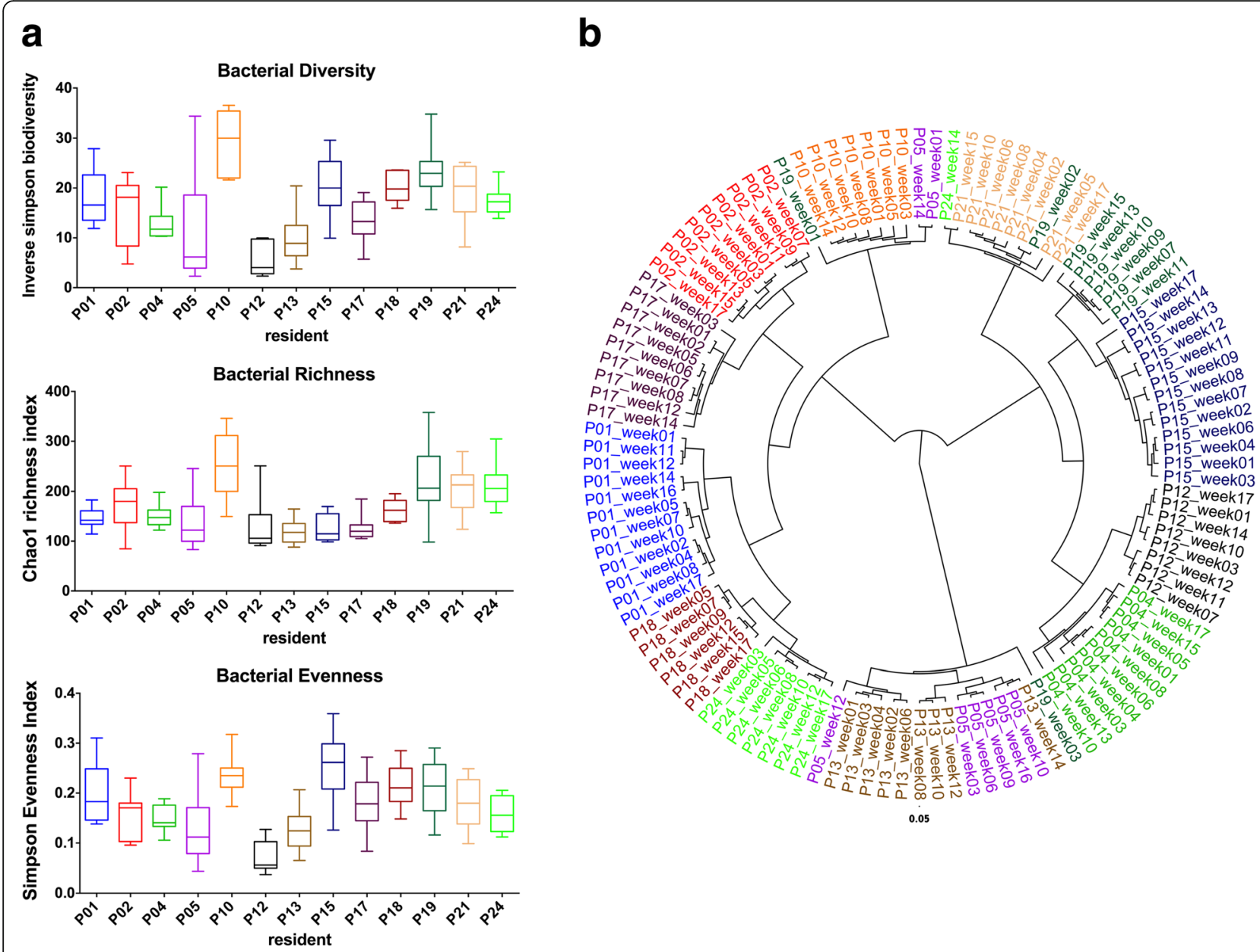

Fig. 2 Species bacterial diversity and species phylotypic tree based on Bray-Curtis distance matrix. a Bacterial diversity (inverse Simpson biodiversity index), bacterial richness (Chao1 richness index) and bacterial evenness (Deduced from Simpson index). Bacterial diversity indexes are expressed as a box plot of the mean from subsampled datasets, whiskers represent minimum and maximum value. Median is shown as a line inside the box. b Phylotype tree of the 118 subsampled datasets built upon a Bray-Curtis distance matrix at the species taxonomical level (average tree is shown, 1000 iterations). The figure also shows all the faecal samples studied by $16 \mathrm{~S}$ rDNA profiling analysis collected from each resident in different weeks

to $C$. difficile without any signs of disease were also detected. Results obtained from PCR-ribotyping and MLVA showed that there was a clonal dissemination within the nursing home residents. Therefore, even if some authors have refuted the theory of person-to person transmission to explain the increase incidence of CDI within hospital awards [22, 23], it seems that in nursing homes the situation is different. Only four different PCR-ribotypes were identified and three of them were toxigenic (UCL16a, UCL46 and 027). Surprisingly, none of them were among the five PCR-ribotypes most commonly identified in Belgian hospitals in 2013 and 2014 [21]. Since 2011, decline in the prevalence of the PCR-ribotype 027 has been reported in different European countries. Furthermore, in Belgium, the proportion of hospitals with the hypervirulent PCR-ribotype 027 decreases from $34 \%$ in 2009 to $15 \%$ in 2013 [20].
Nursing home population is closed and restricted and changes in the prevalence of PCR-ribotypes come later than in hospitals. Therefore, it could be hypothesised that the most prevalent PCR-ribotypes today in hospitals (PCR-ribotypes 078 and 014/020) [21] will be in a few years predominant in nursing homes. In previous studies on elderly gut microbiota, Bacteroidetes and Firmicutes have been reported to dominate, with a marked preponderance of Bacteroidetes over Firmicutes [24-26]. In the present study, the major bacterial phyla identified in residents' microbiota are Firmicutes followed by Bacteroidetes. We also found a higher abundance of Verrucomicrobia than previously observed [27, 28]. The predominance of Firmicutes and Bacteroidetes has also been highlighted in a large cohort study in Belgium [27], although the overall prevalence of Bacteroidetes in our study is higher than the mean value on a large-scale 
Resident

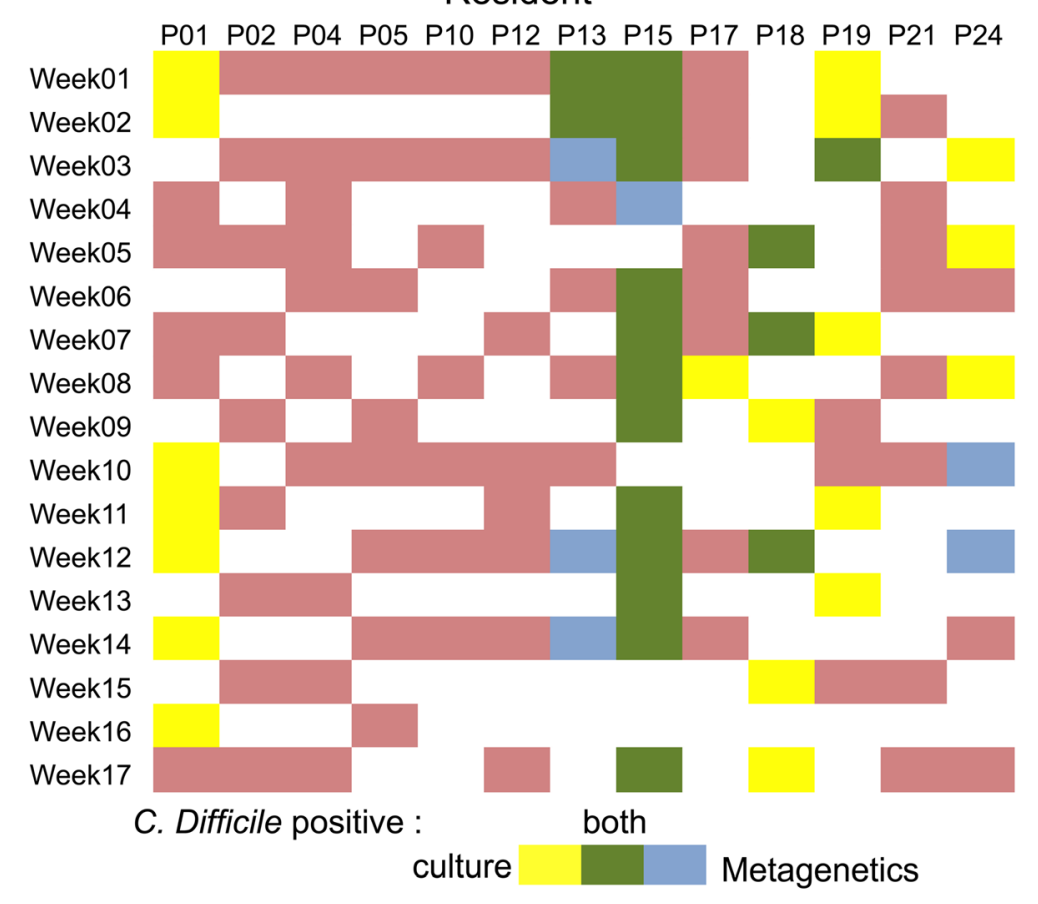

C. Difficile negative :

Fig. 3 C. difficile detection results for the 118 samples analysed by culture and 16S rRNA gene analysis. Grid detailing the detection results for the samples analysed by both methods. For classic microbiology, samples are positive if either direct or enrichment culture is positive. For 165 profiling, samples are positive if at least one sequence read is identical to C. difficile V1-V3 165 rDNA sequence. Red - negative sample; white non-analysed sample; yellow - C. difficile positive culture; blue - C. difficile sequence detection and green - C. difficile positive for both methods

population level (34\% in our study vs $25 \%$ in the Belgian Flemish Gut Flora Project, unpublished data). This increase in Bacteroidetes relative proportion in elderly gut microbiota compared to a matched cohort of younger adults has already been described [27].

It has been recently underlined that longitudinal survey of microbiota in elderly and long-stay residents did not support a model of unstable microbiota and diversity [28]. The longitudinal analysis of the bacterial diversity of community composition showed that bacterial diversity and richness is variable between residents but did not reveal any evolution during the study. Moreover, inter-individual microbiota variability is known to be greater than temporal variability [25] and has been confirmed by community structure analysis.

There are a growing number of publications on the gut microbiota exploration and CDI. Some of them focus on the idea that commensal bacterial populations can protect from CDI [29]. Although no candidate population has emerged, loss of some bacterial genera like Bacteroides has been associated with CDI [30]. Other studies on hospitalized CDI patients described a significant alteration of gut microbiota during CDI along with decreased biodiversity and richness [29, 30]. This alteration includes a rise in Proteobacteria and a decrease in Lachnospiraceae and other butyrate-producing bacteria [29]. However, it should be noted that these alterations do not appear to be specific to CDI and are also observed in patients without $C$. difficile diarrhoea. In a first extensive study on elderly and CDI, Rea et al. [30] showed that there was little difference regarding the microbiota composition between CDI subjects and asymptomatic $C$. difficile carriers. Moreover, only minor bacterial taxon showed a statistically different abundance between $C$. difficile positive subjects and negative individuals.

The 16S rRNA profiling has been performed on a limited cohort of $C$. difficile negative and positive residents. Even if it was longitudinal, we did not focus on the pathology or on the antibiotic use that might have occurred during the survey. We centred this analysis on the hypothesis that in these long term stay residents, $C$. difficile persistent or recurrent colonisation might be associated with more pronounced differences in microbiota between both groups. Significant changes have been observed in $C$. difficile positive individuals in the relative abundance of bacterial populations, but these are limited to the Lachnospiraceae and Verrucomicrobiaceae. Surprisingly, 


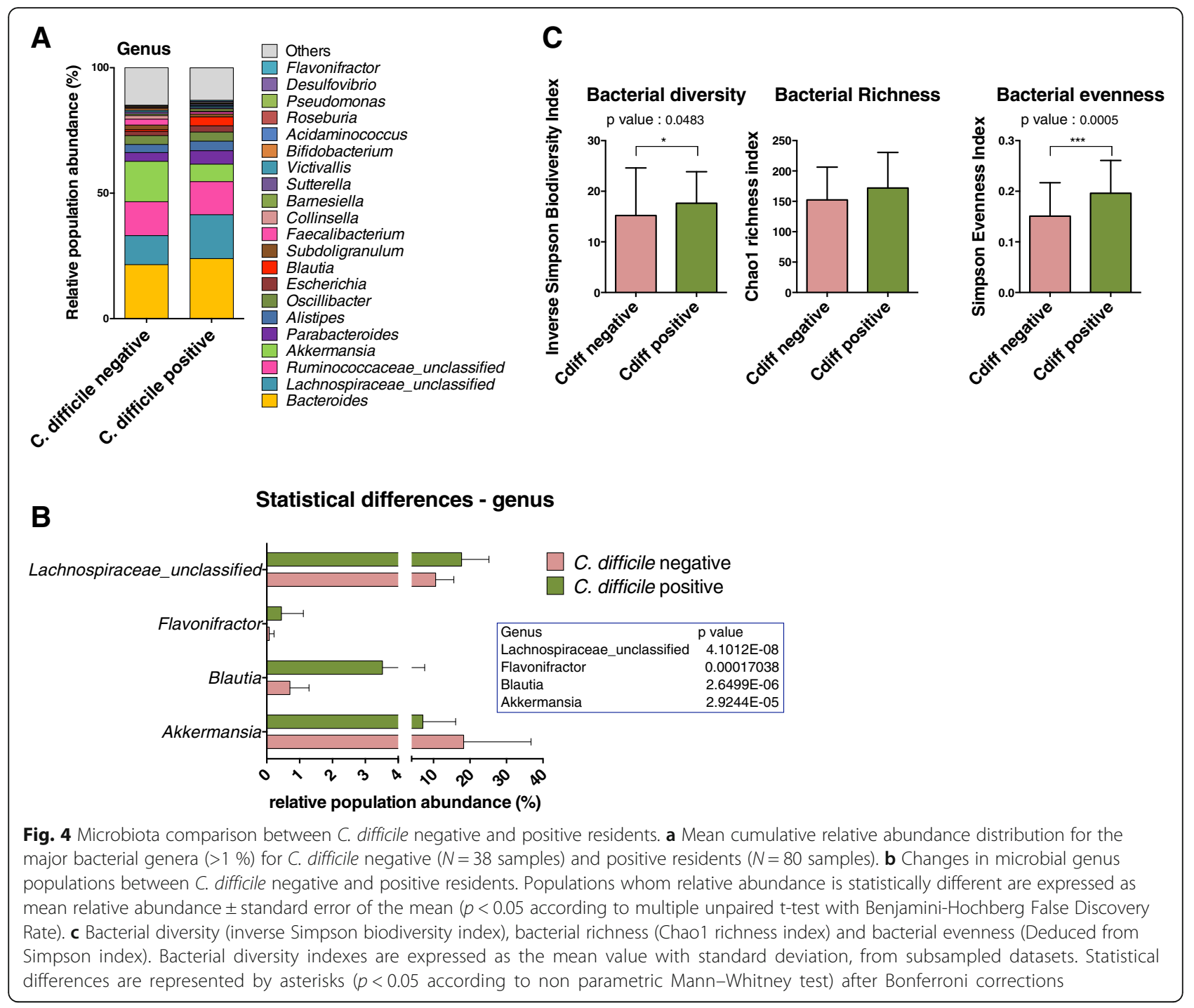

Lachnospiraceae and specifically genus Blautia abundance is higher in $C$. difficile positive individuals, which is quite different from previous reports [31, 32]. Even if CDI diagnosis was not specifically performed during the study, we further split both residents groups regarding the presence of diarrhoeic faeces and observed that this bacterial family abundance is significantly higher in C. difficile positive residents having diarrhoeic faeces compared to diarrhoeic $C$. difficile negative individuals. Verrucomicrobiaceae (genus Akkermansia) is known to be linked to gut health and its abundance seems to be reduced in context of gut inflammation [33]. In addition, Akkermansia is an appealing candidate to become a human probiotic, selected based on established mechanisms of preventive treatment of obesity and diabetes [34, 35]. Even if gut inflammatory status of the residents has not been investigated, it is a known risk factor for $C$. difficile colonisation and could therefore be responsible for this negative correlation.
Positive C. difficile status is not associated with microbiota richness or biodiversity reduction in our study (failed significance after Bonferroni corrections). It appears that impact on gut microbiota structure is associated with actual diarrhoeic episodes instead of $C$. difficile positive status [30]. Recent studies have demonstrated that stool consistency is a dominant factor associated with microbiota composition and negatively correlate species richness with stool looseness [27, 34]. Comparison between diarrhoeic samples versus nondiarrhoeic samples in this study confirms this with a slight yet significant decrease in bacterial richness $(p<0.05$ data not shown) but not in biodiversity.

This study underlines that $C$. difficile status in longterm stay elderly resident is associated with specific changes in microbiota composition. We confirm that the detection alone of this bacterium cannot be linked to major changes in microbiota structure. We have shown that longitudinal study underlines the dynamic of $C$. 
difficile status and the relative stability of gut microbiota in these elderly populations. The major limitation of this is the relatively low number of volunteers. Microbiota analysis has been marked by a strong inter-individual variability, which can certainly influence comparisons between $C$. difficile negative and positive groups. It has been recently shown that several types of microbiota composition might increase susceptibility to CDI [31]. Further studies on long-term stay residents will be needed to improve our knowledge of the $C$. difficile reservoir and susceptibility in nursing homes.

\section{Conclusions}

C. difficile colonisation is higher in nursing homes than in hospitals, with a predominance of the hypervirulent PCR-ribotype 027. MLVA reveals a clonal dissemination of this PCR-ribotype among nursing home residents. In the last years the prevalence of this type is decreasing in hospitals, suggesting that the isolation of elderly in nursing homes has an important impact on the type of strains found. Changes were observed in $C$. difficile positive individuals in the relative abundance of some bacterial populations, including Lachnospiraceae and Verrucomicrobiaceae. Lachnospiraceae and specifically genus Blautia abundance is higher in C. difficile positive individuals than in negative individuals. Positive C. difficile status is not associated with microbiota richness or biodiversity reduction in this study. Notably, a decrease of Akkermansia in positive subjects was repeatedly found. The link between Akkermansia, gut inflammation and $C$. difficile colonisation merits further investigations.

\section{Methods}

\section{Resident recruitment and sampling}

The study was conducted at the Saint-Joséphine (ACIS) nursing home, in the province of Liège (Theux), Belgium. This local nursing home has a total capacity of 110 beds with a total of 73 employees. Data collected included gender, age, clinical status, medical history, recent history of diarrhoea, recent hospitalization, medication, including non-steroidal anti-inflammatory drugs (NSAIDs) or antibiotics, probiotics and changes in diet.

During a 4-month period, from March through June 2013, stool samples from a group of 23 elderly care home residents were collected weekly. Most of the subjects were aged 65 years and older. Faecal sampling was performed from Thursday until early Friday. Two samples per person were collected. The first sample was collected in an individual identified sterile $50 \mathrm{ml}$ tube for further culture to detect $C$. difficile. The second one was collected using the Stool DNA Stabilizer $\left(\mathrm{PSP}^{\mathrm{R}}\right.$ Spin Stool DNA Plus Kit 00310; Invitek, Westburg b.v., Netherlands) to study the microbial biodiversity of the faeces content by amplicon sequencing. Samples obtained were scored as normal, diarrhoea or bloody diarrhoea faeces. They were kept at $4{ }^{\circ} \mathrm{C}$ for a maximum of $48 \mathrm{~h}$ until their arrival in the laboratory for immediate culture or DNA extraction.

\section{C. difficile culture, identification and characterisation}

Culturing of faeces (with and without a phase of enrichment), isolation and identification of $C$. difficile colonies were performed as previously described [36]. Toxin activity of the isolated strains was confirmed by a cytotoxicity assay using confluent monolayer MRC- 5 cells as described previously [37].

\section{Molecular typing of $C$. difficile isolates}

C. difficile isolates were tested using Genotype Cdiff system (Hain Lifescience, Nehren, De) for the presence of the tpi gen, toxin genes $t c d A, t c d B, c d t A$ and $c d t B$, deletions in the regulator gene $t c d C$ and $g y r A$ mutation, according to the manufacturer's instructions.

PCR-ribotyping was performed using the primers and conditions described by Bidet et al. [38]. An international number was used for $C$. difficile strains that presented a PCR-ribotype profile matching the Cardiff ribotypes from the strain collection available in our laboratory. Otherwise, strains were identified with an internal nomenclature.

\section{MLVA}

The DNA extraction was performed using a chelex 100 solution $5 \%$ (Biorad, Nazareth, Be) as described previously [39]. For MLVA, seven VNTR loci (A6, B7, C6, E7, G8, CDR5, CDR60) were studied as previously described [40]. Isolates with MLVA STRD $\leq 2$ were indicative of a high degree of genetic relatedness [41].

\section{S rRNA pyrosequencing and data analysis}

Total bacterial DNA was extracted from the stool samples with the PSP ${ }^{\circ}$ Spin Stool DNA Plus Kit 00310 (Invitek), following the manufacturer's recommendations. 16S rRNA profiling, targeting V1-V3 hypervariable region and sequenced on Roche GS Junior was performed as described previously [36]. Briefly, libraries from 20 samples were run in the same titanium pyrosequencing reaction using Roche multiplex identifiers, and amplicons were sequenced using the Roche GS-Junior Genome Sequencer instrument (Roche). A total of six sequencing runs were necessary to obtain the data for the 118 samples.

Sequence reads processing was treated as previously described [37] using respectively MOTHUR software package v1.35, Pyronoise algorithm and UCHIME algorithm for alignment and clustering, denoising and chimera detection (MOTHUR script has been added as Additional file 6) [42-44]. 16S rRNA Reference alignment and 
taxonomical assignation in MOTHUR were based upon the SILVA database (v1.15) of full-length 16S rRNA sequences [45]. Clustering distance of 0.03 was used for OTU generation. Subsample datasets were obtained and used to evaluate ecological indicators, Richness estimation (Chao1 estimator), microbial biodiversity (reciprocal Simpson index), and the population evenness (derived from Simpson index) at the phylotype species level using MOTHUR. Population structure and community membership were assessed with MOTHUR using distance matrice based on Bray-Curtis dissimilarity index (a measure of community structure which considers shared OTUs and their relative abundances $[46,47]$ abundances).

Weighted UNIFRAC test implemented in MOTHUR v1.35 was used to assess differences regarding bacterial community structure between residents. Statistical differences in bacterial biodiversity, richness and evenness between residents and between $C$. difficile positive and C. difficile negative groups were respectively assessed using one way-ANOVA and Mann-Whitney test using PRISM 6 (Graphpad Software). In order to highlight statistical differences in the bacterial population abundance between groups, multiple unpaired $t$-test with Benjamini-Hochberg False Discovery Rate were performed using PRISM 6 (Graphpad Software). Differences were considered significant for a $p$-value of less than 0.05, adjusted with Bonferroni corrections.

\section{Additional files}

Additional file 1: Quality analysis of the 165 rRNA gene analysis for the 118 human faecal samples. (DOCX $40 \mathrm{~kb}$ )

Additional file 2: Taxonomical distribution deduced by $16 \mathrm{~S}$ rRNA profiling. Bart chart detailing the mean cumulated relative abundance of the major genera for each resident. Bart chart detailing the mean cumulated relative abundance of the major genera for each resident. (PNG $3354 \mathrm{~kb}$ )

Additional file 3: Longitudinal distribution of the ecological indicators Bacterial diversity (inverse Simpson biodiversity index), bacterial richness (Chao 1 richness index) and bacterial evenness (Deduced from Simpson index) expressed for each analysed samples. (PNG 4002 kb)

Additional file 4: Unifrac weighted score and significance between patients. UNIFRAC weighted score (W score) and significance for patients clustering based on Bray-Curtis dissimilarity distance matrix. (DOCX 84 kb)

Additional file 5: Relative abundance of Lachnospiraceae between groups of diarrhoeic/non diarrhoeic and C. difficile status. Lachnospiraceae relative abundance is expressed as mean relative abundance \pm standard error of the mean. Different superscript letters correspond to statistical difference according to one way ANOVA with Tukey-Kramer post-hoc test $(p<0.05)$. (PNG $1052 \mathrm{~kb})$

Additional file 6: MOTHUR script used for $16 \mathrm{~S}$ rRNA gene analysis. (TXT $1 \mathrm{~kb}$ )

\section{Abbreviations}

C. difficile: Clostridium difficile; CDI: C. difficile infection; MLVA: Multilocus variable number of tandem repeats analysis; NSAIDS: Non-steroidal antiinflammatory drugs; OTUs: Operational taxon units; STRD: Summed tandem repeat differences; VNTR: Variable number tandem repeat

\section{Acknowledgements}

The authors wish to thank the nursing home Saint-Josephine for participating in this study.

\section{Funding}

This research did nit receive external funding.

\section{Availability of data and materials}

All the biosample raw reads have been deposited at the National Center for Biotechnology Information (NCBI) and are available under de Bioproject PRJNA315622 (http://www.ncbi.nlm.nih.gov/bioproject).

\section{Authors' contributions}

CR, BT and GD have designed the study. CR, BT, NK and PB have performed sampling and sample processing. VA and JVB have contributed towards typing. BT has performed $16 \mathrm{~S}$ rRNA gene analysis. CR, BT, VA, MD and GD have performed data analysis. All authors have participated at writing. All authors have read and approved the final manuscript.

\section{Competing interests}

The authors declare that they have no competing interests.

\section{Consent for publication}

Not applicable.

\section{Ethics approval and consent to participate}

The study was approved by the Hospital-Faculty Ethics Committee of the University of Liège (707). Written informed consent to participate was obtained from all of the participants or their next of kin in case of cognitively impairment.

\section{Author details}

${ }^{1}$ Food Science Department, FARAH, Faculty of Veterinary Medicine, University of Liège, Liège, Belgium. ${ }^{2}$ National Reference Laboratory for Clostridium difficile, Cliniques Universitaires Saint Luc, Microbiology Unit, Catholic University of Louvain, Brussels, Belgium. ${ }^{3}$ Nursing Home Saint-Joséphine site de la Chaussée, ACIS, Theux, Belgium.

Received: 13 April 2016 Accepted: 23 September 2016

Published online: 01 October 2016

\section{References}

1. George RH, Symonds JM, Dimock F, Brown JD, Arabi Y, Shinagawa N, Keighley MR, Alexander-Williams J, Burdon DW. Identification of Clostridium difficile as a cause of pseudomembranous colitis. Br Med J. 1978;1:695.

2. George WL, Sutter VL, Goldstein EJ, Ludwig SL, Finegold SM. Aetiology of antimicrobial-agent-associated colitis. Lancet. 1978;1:802-3.

3. Larson HE, Price AB, Honour P, Borriello SP. Clostridium difficile and the aetiology of pseudomembranous colitis. Lancet. 1978;1:1063-6.

4. Kuijper EJ, Barbut F, Brazier JS, Kleinkauf N, Eckmanns T, Lambert ML, et al. Update of C. difficile infection due to PCR-ribotype 027 in Europe, 2008. Euro Surveill. 2008;13:31.

5. Barbut F, Jones G, Eckert C. Epidemiology and control of C. difficile infections in healtcare settings: an update. Curr Opin Infect Dis. 2011;24:370-6.

6. Nanwa N, Kendzerska T, Krahn M, Kwong JC, Daneman N, Witteman W, et al. The economic impact of Clostridium difficile infection: a systematic review. Am J Gastroenterol. 2015;110:511-9.

7. Jones AM, Kuijper EJ, Wilcox MH. Clostridium difficile: a European perspective. J Infect. 2013;66:115-28.

8. Rodriguez C, Korsak N, Taminiau B, Avesani V, Van Broeck J, Delmée M, Daube G. Clostridium difficile infection in elderly nursing home residents. Anaerobe. 2014;30:184-7.

9. Hensgens M, Dekkers OM, Goorhuis A, Lecessie S, Kuijper EJ. Predicting a complicated course of Clostridium difficile infection at the bedside. Clin Microbiol Infect. 2014;20:0301-8.

10. Kelly CP. Can we identified patients at high risk of recurrent Clostridium difficile infection? Clin Microbiol Infect. 2012;18:21-7.

11. Theriot CM, Young VB. Microbial and metabolic interactions between the gastrointestinal track and Clostridium difficile infection. Gut Microbes. 2014;5:86-95. 
12. Rodriguez C, Taminiau B, Van Broeck J, Delmée M, Daube G. Clostridium difficile infection and intestinal microbiota interactions. Microb Pathog. 2015;89:201-9.

13. Arvand M, Moser V, Schwehn C, Bettge-Weller G, Hensgens MP, Kuijper EJ. High prevalence of Clostridium difficile colonisation among nursing home residents in Hesse. Germany PlosOne. 2012;7:e30183.

14. Mylotte JM, Russell S, Sackett B, Vllone M, Antelek M. Surveillance for Clostridium difficile infection in nursing homes. J Am Geriatr Soc. 2013:61:122-5.

15. Hopkins MJ, Sharp R, Macfarlane GT. Variation in human intestinal microbiota with age. Dig Liver Dis Off J Ital Soc Gastroenterol Ital Assoc Study Liver. 2002;34 Suppl 2:S12-8.

16. Marwick CA, Yu N, Lockhart MC, McGuigan CC, Wiuff C, Davey PG, et al. Community associated Clostridium difficile infection among older people in Tayside, Scotland, is associated with antibiotic exposure and care home residence: cohort study with nested case-control. J Antimicrob Chemother. 2013;68:2927-33

17. Ryan J, Murphy C, Twomey C, Ross RP, Rea MC, McSharry J, et al. Asymptomatic carriage of Clostridium difficile in an irish community care institution for the elderly: prevalence and characteristics. Ir J Med Sci. 2010;179:245-50.

18. Stuart RL, Kotsanas D, Webb B, Vandergraaf S, Gillespie EE, Hoog GG, et al. Prevalence of antimicrobial-resistance organisms in residential aged care facilities. Med J Aust. 2011;7:530-3.

19. Simor AE, Yake SL, Tsimidis K. Infection due to Clostridium difficile among elderly residents of a long-term-care facility. Clin Infect Dis. 1993;17:672-8.

20. Neely F, Boudewijn C, Lambert ML. Epidemiology of Clostridium difficile infection in Belgium. Report 2014. ISP-WIW 2014. http://www.nsih.be/ download/CDIF/CDIF-AR-2014-EN.pdf. Accessed 31 Mar 2016.

21. Neely F, Lambert ML. Epidemiology of Clostridium difficile infection in Belgium. Report 2015. ISP-WIW 2015. http://www.nsih.be/download/CDIF/ CDIF-AR-2015-EN.pdf. Accessed 31 Mar 2016

22. Eyre DW, Golubchik T, Gordon NC, Bowden R, Piazza P, Batty EM, et al. A pilot study of rapid benchtop sequencing of Staphylococcus aureus and Clostridium difficile for outbreak detection and surveillance. BMJ Open. 2012:2:e001124.

23. Rodriguez-Palacios A, Borgamann S, Kline TR, LeJeune JT. Clostridium difficile in foods and animals: history and measures to reduce exposure. Anim Health Res Rev. 2013;14:11-29.

24. Claesson MJ, Cusack S, O'Sullivan O, Greene-Diniz R, de Weerd H, Flannery E, et al. Composition, variability, and temporal stability of the intestinal microbiota of the elderly. Proc Natl Acad Sci USA. 2011;108 Suppl 1:4586-91.

25. Claesson MJ, Jeffery IB, Conde S, Power SE, O'Connor EM, Cusack S, et al. Gut microbiota composition correlates with diet and health in the elderly. Nature. 2012;488:178-84

26. Mariat $D$, Firmesse $O$, Levenez F, Guimarăes $V$, Sokol H, Doré J, et al. The firmicutes/bacteroidetes ratio of the human microbiota changes with age. BMC Microbiol. 2009;9:123.

27. Falony $G$, Joossens M, Vieira-Silva S, Wang J, Darzi Y, et al. Population-level analysis of gut microbiome variation. Science. 2016;352:560-4.

28. O'Toole PW, Jeffery IB. Gut microbiota and aging. Science. 2015;350:1214-5.

29. Antharam VC, Li EC, Ishmael A, Sharma A, Mai V, Rand KH, et al. (2013) Intestinal dysbiosis and depletion of butyrogenic bacteria in Clostridium difficile infection and nosocomial diarrhoea. J Clin Microbiol. 2013;51:2884-92.

30. Rea MC, O'Sullivan O, Shanahan F, OToole PW, Stanton C, Ross RP, et al. Clostridium difficile carriage in elderly subjects and associated changes in the intestinal microbiota. J Clin Microbiol. 2012;50:867-75.

31. Schubert AM, Rogers MAM, Ring C, Mogle J, Petrosino JP, Young VB, et al. Microbiome data distinguish patients with Clostridium difficile infection and Non-C. Difficile-associated diarrhoea from healthy controls. MBio. 2014;5:e01021-14

32. Pérez-Cobas AE, Artacho A, Ott SJ, Moya A, Gosalbes MJ, et al. Structural and functional changes in the gut microbiota associated to Clostridium difficile infection. Front Microbiol. 2014:5:335.

33. Derrien M, Belzer C, de Vos WM. Akkermansia muciniphila and its role in regulating host functions. Microb Pathog. 2016;9:1-11.

34. Cany PD, Everard A. Akkermansia muciniphila: a novel target controlling obesity, type 2 diabetes and inflammation? MédSci. 2014;30:125-7.

35. Shin N-R, Lee J-C, Lee H-Y, Kim M-S, Whom TW, Lee M-S, et al. An increase in the Akkermansia spp population induced by metformin treatment improves glucose homeostasis in diet-induced obese mice. Gut. 2014;63:727-35.
36. Rodriguez C, Taminiau B, Brévers B, Avesani V, Van Broeck J, Leroux AA, et al. Carriage and acquisition rates of Clostridium difficile in hospitalized horses, including molecular characterization, multilocus sequence typing and antimicrobial susceptibility of bacterial isolates. Vet Microbiol. 2014;172:309-17

37. Rodriguez C, Taminiau B, Brévers B, Avesani V, Van Broeck J, Leroux A, et al. Faecal microbiota characterisation of horses using rDNA barcoded pyrosequencing, and carriage rate of Clostridium difficile at hospital admission. BMC Microbiol. 2015;15:181.

38. Rodriguez C, Taminiau B, Van Broeck J, Avesani V, Delmée M, Daube G. 2014. Clostridium difficile in young farm animals and slaughter animals in Belgium. Anaerobe. 2014;18:621-5.

39. Bidet P, Barbut F, Lalande V, Burghoffer B, Petit JC. Development of a new PCR-ribotyping method based on ribosomal RNA gene sequencing. FEMS Microbiol Lett. 1999;175:261-6.

40. O'Neill GL, Ogunsola FT, Brazier JS, Duerden BI. Modification of a PCR ribotyping method for application as a routine typing scheme for Clostridium difficile. Anaerobe. 1996;2:205-9.

41. Manzoor SE, Tanner HE, Marriott CL, Brazier JS, Hardy KJ, Platt S, Hawkey PM. Extended multilocus variable-number tandem-repeat analysis of Clostridium difficile correlates exactly with ribotyping and enables identification of hospital transmission. J Clin Microbiol. 2011:49:3523-30.

42. Schloss PD, Westcott SL, Ryabin T, Hall JR, Hartmann M, Hollister EB, et al. Introducing mothur: open-source, platform-independent, communitysupported software for describing and comparing microbial communities. Appl Environ Microbiol. 2009;75:7537-41.

43. Quince C, Lanze'n A, Curtis TP, Davenport RJ, Hall N, Head IM, et al. Accurate determination of microbial diversity from 454 pyrosequencing data. Nat. Methods. 2009;6:639-41.

44. Edgar RC, Haas BJ, Clemente JC, Quince C, Knight R. UCHIME improves sensitivity and speed of chimera detection. Bioinformatics. 2011;27:2194-200.

45. Pruesse E, Quast C, Knittel K, Fuchs BM, Ludwig W, Peplies J, et al. SILVA: a comprehensive online resource for quality checked and aligned ribosomal RNA sequence data compatible with ARB. Nucleic Acids Res. 2007;35:7188-96.

46. Bray JR, Curtis JT. An ordination of upland forest communities of southern Wisconsin. Ecol Monogr. 1957;27:325-49.

47. Hunter PR, Gaston MA. Numerical index of the discriminatory ability of typing systems: an application of Simpson's index of diversity. J Clin Microbiol. 1988:26:2465-6.

\section{Submit your next manuscript to BioMed Central and we will help you at every step:}

- We accept pre-submission inquiries

- Our selector tool helps you to find the most relevant journal

- We provide round the clock customer support

- Convenient online submission

- Thorough peer review

- Inclusion in PubMed and all major indexing services

- Maximum visibility for your research

Submit your manuscript at www.biomedcentral.com/submit

) Biomed Central 